\title{
Numerical continuation of families of heteroclinic connections between periodic orbits in a Hamiltonian system
}

\author{
E. Barrabés ${ }^{1}$, J. M. Mondelo ${ }^{2}$ and M. Ollée ${ }^{3}$ \\ ${ }^{1}$ Dept. Informàtica, Matemàtica Aplicada i Estadística, Universitat de Girona, \\ Avda. Lluís Santaló s/n, 17071 Girona, Spain \\ 2 IEEC \& Dept. Matemàtiques. Universitat Autònoma de Barcelona, Edifici C, \\ 08193 Bellaterra, Spain. \\ ${ }^{3}$ Dept. de Matemàtica Aplicada I. Universitat Politècnica de Catalunya, Diagonal \\ 647, 08028 Barcelona, Spain.
}

E-mail: barrabes@imae.udg.edu, jmm@mat.uab.es, merce.olle@upc.edu

\begin{abstract}
This paper is devoted to the numerical computation and continuation of families of heteroclinic connections between hyperbolic periodic orbits of a Hamiltonian system. We describe a method that requires the numerical continuation of a nonlinear system that involves the initial conditions of the two periodic orbits, the linear approximations of the corresponding manifolds and a point in a given Poincaré section where the unstable and stable manifolds match. The method is applied to compute families of heteroclinic orbits between planar Lyapunov periodic orbits around the collinear equilibrium points of the Restricted Three-Body Problem in different scenarios. In one of them, for the Sun-Jupiter mass parameter, we provide ranges of energy for which the transition between different resonances is possible.
\end{abstract}

AMS classification scheme numbers: 70F07, 70F15, 70H12, 70H33, 70K44 


\section{Introduction}

Homoclinic and heteroclinic connections of hyperbolic invariant sets play an important role in the study of dynamical systems from a global point of view. The field of astrodynamics provides a nice example of their application. In the dynamical models used for preliminary mission design $₫$ homoclinic and heteroclinic phenomena, together with their associated chaotic behaviour, arise in situations in which the gravitational pulls from different bodies on a spacecraft compete in equality of conditions (contrary e.g. to a planetary flyby, in which case a two body approximation is sufficient for mission design purposes). This is the case of libration point (LP) missions, for which dynamical systems techniques were introduced relatively recently and are now widely spread $[7,8,10,11,14,22]$. Genesis [14] has been the first LP mission to make use of a heteroclinic connection between objects related to the $L_{1}$ and $L_{2}$ points of the Sun-Earth system. More recently, Artemis [22] has also used heteroclinic connections between the $L_{1}$ and $L_{2}$ dynamics but in the Earth-Moon system, with a more complex final trajectory. The trajectories of these two missions are shown in Figure 1. The usability of homoclinic and heteroclinic phenomena for the design of LP missions is limited to the thoroughness of current numerical studies, as well as to the numerical methodology available to perform them in a manner as systematic as possible. The main motivation of this paper is to contribute to these two aspects.

Subfamilies (in terms of the energy ranges covered) of the families of heteroclinic connections continued in this paper have been addressed in previous works, both analytically and numerically. Having as main motivation the explanation of resonance transitions observed in the comet Oterma, in [18] the authors numerically compute an heteroclinic connection between Lyapunov orbits around $L_{1}$ and $L_{2}$ which, combined with previous analytical results on homoclinic connections [19, 20], allow them to both explain comet Oterma's behaviour and construct trajectories with prescribed itineraries via symbolic dynamics. Other works $[4,9,12]$ compute heteroclinic connections through the use of semianalytical techniques (asymptotic expansions with coefficients computed in finite-precision arithmetic). In these works, individual connections are typically found by matching the corresponding manifolds on a Poincaré section, and families are described by individually repeating this matching process for several values of the continuation parameter. There are also works based on rigorous numerics [24, 25] which provide proofs on the existence of some connections between periodic orbits.

This paper naturally extends the methodology introduced in [3] to the case of heteroclinic connections of periodic orbits (p.o.). As in that reference, the numerical method proposed for the continuation of families of heteroclinic connections of p.o. consists of solving a nonlinear system of equations whose solution is a curve

$\ddagger$ This is the phase in which several candidate trajectories are analysed in an approximate model, in order to choose the one that will be numerically refined to a high-fidelity model and actually used. The approximate model should be accurate enough for the considered trajectories to preserve their properties when refined to the high-fidelity model. 

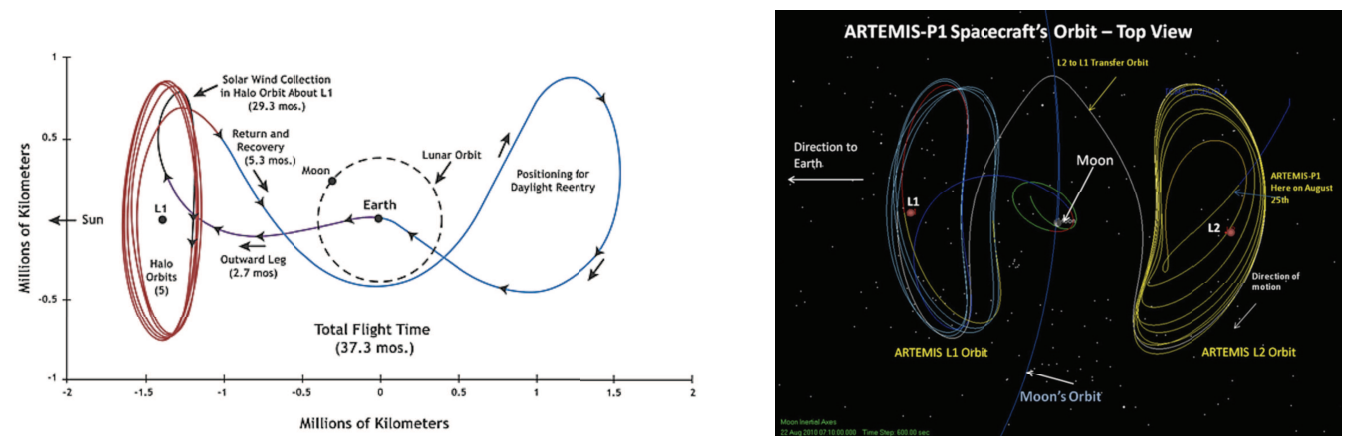

Figure 1. Left: Projection in the rotating $(x, y)$ plane of the trajectories of two missions: the Genesis mission in the Sun-Earth system (left) and the Artemis spacecraft in the Earth-Moon system (right). (Downloaded from NASA's official web pages)

that corresponds to the family. This system includes all the necessary ingredients to compute such a family: the equations for two different p.o., the eigenvalue/eigenvector equations for the linear approximations of the corresponding invariant manifolds of the p.o., and matching conditions for the manifolds in a section. The family of solutions of the system of equations is numerically continued by a standard predictor-corrector method (see, e.g. [2]). Both the system of equations and its differential with respect to the unknowns are evaluated by direct numerical integration of the differential equations, together with their first and second variational equations. The instability due to the hyperbolic character of all the p.o. considered is coped with a multiple shooting strategy.

Compared to previous methodology, the procedure presented here is intended to address two drawbacks. On the one hand, to overcome the convergence restrictions of the asymptotic expansions used in semianalytical techniques. On the other hand, to automate the process of computation of families of heteroclinic connections (it is not necessary to repeat the matching computations to get an heteroclinic connection at each energy level). It must be noted that some previous works (e.g. [9, 12, 6]) have been able to obtain heteroclinic connections of tori through the use of semianalytical techniques. The numerical procedure presented here has a natural extension to connections of tori (or even p.o. and tori), but its implementation has not been attempted yet and is work in progress.

Although the methodology presented here is valid for any Hamiltonian system, all the computations presented in this paper correspond to the Circular Restricted Three-Body Problem (CRTBP), and for simplicity we have restricted ourselves to the continuation of heteroclinic orbits between planar Lyapunov p.o. around the collinear equilibrium points $L_{i}, i=1,2,3$. A first illustration of the procedure is given by extending some of the computations in [4], that correspond to heteroclinic connections between p.o. around the $L_{1,2}$ points of the Earth-Moon system. As a second application, the family of the heteroclinic connections found in [18] is continued, providing a range of energies for which the transition from the exterior 2:3 resonance to the interior 3:2 resonance in the Sun-Jupiter system is possible. The study is done systematically, and 
provides other resonances that can also be connected through the family of heteroclinic connections mentioned. As a final application, a value of the CRTBP mass parameter is found for which there exists two families of heteroclinic connections between Lyapunov orbits around $L_{2}$ and $L_{3}$, and these families are followed.

The paper is organised as follows: in Section 2 we describe the numerical method for the continuation of heteroclinic orbits between p.o. in the general setting of a given Hamiltonian of $n$ degrees of freedom. In Section 3 we briefly summarise the main features of the CRTBP. Section 4 presents the numerical explorations previously described. The paper is ended with some concluding remarks.

\section{Continuation of families of heteroclinic connections between periodic orbits. Numerical methodology}

Consider a Hamiltonian system with Hamiltonian function $H(x)$ and equations

$$
\dot{x}=Z_{H}(x),
$$

for $x \in \mathbb{R}^{2 n}$, and denote by $\phi_{t}(x)$ the time- $t$ flow associated to the equations. Denote also by $h$ the constant value of the Hamiltonian $H$ along a solution, which is known as the energy of the orbit.

An heteroclinic connection between two invariant objects $X, Y$ is a trajectory that tends backward in time to $X$ and forward in time to $Y$, so it lies in $W^{u}(X) \cap W^{s}(Y)$, where $W^{u}(X)$ and $W^{s}(Y)$ stand for the unstable and stable manifolds of $X$ and $Y$, respectively. Consider a p.o. $X$ with hyperbolic character, and assume that we have a parametrisation of its $2 \mathrm{D}$ unstable and stable manifolds that can be numerically evaluated. We denote these parameterisations as $\psi^{u}(\theta, \xi)$ and $\psi^{s}(\theta, \xi)$, where $\theta$ is an angle and $\xi \in \mathbb{R}$ (we give actual formulae later in this Section). We also assume that $\psi^{u / s}(\theta, \xi)$ describes the p.o. for $\xi=0$, and that the two branches of the manifold are given by $\xi>0$ and $\xi<0$.

Let $\Sigma=\{g(x)=0\}$ be a hypersurface known to be intersected by the invariant manifolds, where $g: \mathbb{R}^{2 n} \rightarrow \mathbb{R}$ is a function defined on a neighbourhood of the intersection. Given a point $x$, we consider two associated Poincaré maps: $P_{\Sigma}^{+}$, which propagates the flow (starting at $x$ ) forward in time until some intersection with $\Sigma$ (more than one cut may be necessary), and $P_{\Sigma}^{-}$, which does the same backward in time. Given two hyperbolic p.o. $X_{1}$ and $X_{2}$, with $\psi_{1}^{u}$ and $\psi_{2}^{s}$ the corresponding parametrisations of $W^{u}\left(X_{1}\right)$ and $W^{s}\left(X_{2}\right)$, we can choose a value $\xi_{0}$, with $\left|\xi_{0}\right|$ small, and consider the function

$$
F\left(\theta^{u}, \theta^{s}\right)=P_{\Sigma}^{+}\left(\psi_{1}^{u}\left(\theta^{u}, \xi_{0}\right)\right)-P_{\Sigma}^{-}\left(\psi_{2}^{s}\left(\theta^{s}, \xi_{0}\right)\right) .
$$

The specific values of $\left(\theta^{u}, \theta^{s}\right)$ for which $F\left(\theta^{u}, \theta^{s}\right)$ is zero correspond to a heteroclinic connection. In particular, the points $\psi_{1}^{u}\left(\theta^{u}, \xi_{0}\right)$ (which is close to the p.o. $X_{1}$ in $W^{u}\left(X_{1}\right)$ ), $\psi_{2}^{s}\left(\theta^{s}, \xi_{0}\right)$ (also close to the p.o. $X_{2}$ in $\left.W^{s}\left(X_{2}\right)\right)$, and $P^{+}\left(\psi_{1}^{u}\left(\theta^{u}, \xi_{0}\right)\right)=P^{-}\left(\psi_{2}^{s}\left(\theta^{s}, \xi_{0}\right)\right)$ (which is in $\Sigma$ ), belong to the heteroclinic orbit. 
The function $F$ and its differential can be numerically evaluated by numerical integration of the system of ODE of the problem and its first variational equations, so Newton's method can be used to find roots of $F$. In order to obtain initial conditions for the iteration process, it is convenient to display the intersection of each manifold with the section $\Sigma,\left\{P_{\Sigma}^{+}\left(\psi_{1}^{u}\left(\theta, \xi_{0}\right)\right)\right\}_{\theta \in[0,2 \pi)}$ and $\left\{P_{\Sigma}^{-}\left(\psi_{2}^{s}\left(\theta, \xi_{0}\right)\right)\right\}_{\theta \in[0,2 \pi)}$. Although the section $\Sigma$ should be defined locally, in practice, it is more convenient to work with global sections defined by an implicit equation $\{g(x)=0\}$ (actually, in all the computations we have used just hyperplanes). Since the manifolds are 2D tubes, each intersection is a $1 \mathrm{D}$ set. Each common point of these two sets belongs to a heteroclinic connection, and therefore corresponds to a zero of $F$.

For the evaluation of $\psi_{1}^{u}, \psi_{2}^{s}$, we use the linear approximation of an invariant manifold of a p.o. $X$. Assume that $x_{0}$ is an initial condition of a $T$-p.o., so that $\phi_{T}\left(x_{0}\right)=x_{0}$. Then the p.o. can be parametrised by an angle as

$$
\varphi(\theta)=\phi_{\frac{\theta}{2 \pi} T}\left(x_{0}\right),
$$

for $\theta \in[0,2 \pi]$. Assume that $\Lambda>0$ is an eigenvalue of the monodromy matrix $D \phi_{T}\left(x_{0}\right)$ ( $\Lambda>1$ for the unstable manifold, $\Lambda<1$ for the stable one), and $v_{0} \in \mathbb{R}^{2 n}$ is a corresponding eigenvector. Define

$$
v(\theta)=\Lambda^{-\theta / 2 \pi} D \phi_{\frac{\theta}{2 \pi} T}\left(x_{0}\right) v_{0}
$$

for $\theta \in[0,2 \pi]$, which is the eigenvector associated to the point $\varphi(\theta)$. Then

$$
\bar{\psi}(\theta, \xi)=\varphi(\theta)+\xi v(\theta) \text {. }
$$

for $\theta \in[0,2 \pi]$ and $\xi \in \mathbb{R}$, gives the linear approximation of the invariant manifold, which needs $|\xi|$ to be small enough in order to be accurate. A first-order Taylor expansion shows that, for bounded $|t|$,

$$
\phi_{t}(\bar{\psi}(\theta, \xi))=\bar{\psi}\left(\theta+t \omega, e^{t \lambda} \xi\right)+O\left(\xi^{2}\right)
$$

for $\omega=2 \pi / T, \lambda=\omega \ln \Lambda /(2 \pi)$. In all the computations done throughout this paper we have taken $|\xi|$ of the order of $10^{-6}$.

For the continuation of families of heteroclinic connections, we can consider the energy $h$ as a continuation parameter. This means that, for each value of the energy, we would need to compute the corresponding two p.o., their eigenvalues and eigenvectors, and a new initial seed in order to solve the equations $F\left(\theta^{u}, \theta^{s}\right)=0$, with $F$ defined as in (1). We would like to prevent this by performing continuation on Equation (1) through a standard predictor-corrector method (see e.g. [2]), in order to obtain larger continuation steps and not to worry about turning points. The problem with Equation (1) is that the evaluation of $\psi_{1}^{u}, \psi_{2}^{s}$ depends on the eigenvalues and eigenvectors of the monodromy matrices of $X_{1}, X_{2}$. In order to perform continuation, they would need to be differentiated with respect to the initial conditions of $X_{1}, X_{2}$. Instead, it is more convenient to add the eigenvalue and eigenvector conditions to the continuation equations, plus normalisation conditions in order to have local uniqueness of the eigenvectors. In this way, we can lay out a whole (nonlinear) system whose solution gives both the LPO and the heteroclinic connection. 
In order to write this system of equations explicitly, let $h \in \mathbb{R}$ be an energy level, $x_{1}, x_{2} \in \mathbb{R}^{2 n}$ initial conditions of the LPO $X_{1}$ and $X_{2}$ of periods $T_{1}, T_{2}$ respectively, $\Lambda^{u} \in \operatorname{Spec} D \phi_{T_{1}}\left(x_{1}\right)$, with $\Lambda^{u}>1, \Lambda^{s} \in \operatorname{Spec} D \phi_{T_{2}}\left(x_{2}\right)$, with $0<\Lambda^{s}<1$, and $v^{u}, v^{s}$ the corresponding eigenvectors. Consider also $\theta^{u}, \theta^{s} \in[0,2 \pi]$ starting phases on the linear approximation of the unstable and stable manifolds, respectively. Let $T^{u}, T^{s} \in \mathbb{R}$ be the times to intersect the section $\Sigma$ from the starting points $\psi_{1}^{u}\left(\theta^{u}, \xi\right), \psi_{2}^{s}\left(\theta^{s}, \xi\right)$ on the (linear approximation of the) unstable and stable manifolds, respectively (for a given $\xi$ ). The goal of the introduction of $T^{u}, T^{s}$ is to avoid the need to take into account the number of cuts with the sections, which is inconvenient but necessary if we allow Poincaré maps to appear explicitly in the continuation equations, as happens in Equation (1).

We also consider $p: \mathbb{R}^{2 n} \longrightarrow \mathbb{R}$ a function defining a Poincaré section for the p.o., and $g: \mathbb{R}^{2 n} \longrightarrow \mathbb{R}$ a function defining the Poincaré section to match the manifolds, this is, $\Sigma=\{g(x)=0\}$. The system of equations used for the continuation of heteroclinic connections between p.o. is

$$
\begin{aligned}
H\left(x_{1}\right)-h & =0, \\
p\left(x_{1}\right) & =0, \\
\phi_{T_{1}}\left(x_{1}\right)-x_{1} & =0, \\
H\left(x_{2}\right)-h & =0, \\
p\left(x_{2}\right) & =0, \\
\phi_{T_{2}}\left(x_{2}\right)-x_{2} & =0, \\
\left\|v^{u}\right\|^{2}-1 & =0, \\
D \phi_{T_{1}}\left(x_{1}\right) v^{u}-\Lambda^{u} v^{u} & =0, \\
\left\|v^{s}\right\|^{2}-1 & =0, \\
D \phi_{T_{2}}\left(x_{2}\right) v^{s}-\Lambda^{s} v^{s} & =0, \\
g\left(\phi_{T^{u}}\left(\psi_{1}^{u}\left(\theta^{u}, \xi_{0}\right)\right)\right) & =0, \\
g\left(\phi_{T^{s}}\left(\psi_{2}^{s}\left(\theta^{s}, \xi_{0}\right)\right)\right) & =0, \\
\phi_{T^{u}}\left(\psi_{1}^{u}\left(\theta^{u}, \xi_{0}\right)\right)-\phi_{T^{s}}\left(\psi_{2}^{s}\left(\theta^{s}, \xi_{0}\right)\right) & =0,
\end{aligned}
$$

where $\xi_{0}$ is kept fixed to a small value (usually $10^{-6}$ ). The unknowns are

$$
h, x_{1}, x_{2}, T_{1}, T_{2}, \Lambda^{u}, v^{u}, \Lambda^{s}, v^{s}, \theta^{u}, T^{u}, \theta^{s}, T^{s} .
$$

Here $T_{1}, T_{2}, \theta^{u}, T^{u}>0$ and $\theta^{s}, T^{s}<0$.

In the computations of Section 4 we often deal with highly unstable p.o. $\left(\Lambda^{u} \gg\right.$ $1 \gg \Lambda^{s}>0$ ), and also the integration times $T^{u}, T^{s}$ become large. Not only to maintain high precision, but also to guarantee convergence when solving the system (2), is it necessary to use multiple shooting. We have implemented it both in the p.o. and the connections. More precisely, we have added new points as unknowns, $x_{1}^{1}, \ldots, x_{m_{1}-1}^{1}$ along the p.o. $X_{1}, x_{1}^{2}, \ldots, x_{m_{2}-1}^{2}$ along the p.o. $X_{2}, z_{1}^{u}, \ldots, z_{m^{u}-1}^{u}$ along the unstable branch, and $z_{1}^{s}, \ldots, z_{m^{s}-1}^{s}$ along the stable one. The corresponding matching equations 
have been added to system (2), namely

$$
\begin{aligned}
\phi_{T_{i}^{1}}\left(x_{i}^{1}\right)-x_{i+1}^{1}=0, & i=0, \ldots, m_{1}-2, \quad \phi_{T_{m_{1}-1}^{1}}\left(x_{m_{1}-1}^{1}\right)-x_{0}^{1}=0, \\
\phi_{T_{i}^{2}}\left(x_{i}^{2}\right)-x_{i+1}^{2}=0, & i=0, \ldots, m_{2}-2, \quad \phi_{T_{m_{2}-1}^{2}}\left(x_{m_{2}-1}^{2}\right)-x_{0}^{2}=0, \\
\phi_{T_{i}^{u}}\left(z_{i}^{u}\right)-z_{i+1}^{u}=0, & i=0, \ldots, m^{u}-2, \quad g\left(\phi_{T_{m}^{u}-1}^{u}\left(z_{m^{u}-1}^{u}\right)\right)=0, \\
\phi_{T_{i}^{s}}^{u}\left(z_{i}^{s}\right)-z_{i+1}^{s}=0, & i=0, \ldots, m^{s}-2, \quad g\left(\phi_{T_{m^{s}-1}^{s}}^{s}\left(z_{m^{s}-1}^{s}\right)\right)=0,
\end{aligned}
$$

with $x_{0}^{1}=x_{1}, x_{0}^{2}=x_{2}, z_{0}^{u}=\psi_{1}^{u}\left(\theta^{u}, \xi_{0}\right)$ and $z_{0}^{s}=\psi_{2}^{s}\left(\theta^{s}, \xi_{0}\right)$. In the continuations of Section 4 it often happens that convergence becomes poor. Except in the cases that some trajectory is approaching collision, good convergence is recovered by increasing the number of intermediate points $m_{1}, m_{2}, m^{u}, m^{s}$, and recomputing the integration times $\left\{T_{i}^{1}\right\}_{i},\left\{T_{i}^{2}\right\}_{i},\left\{T_{i}^{u}\right\}_{i},\left\{T_{i}^{s}\right\}_{i}$.

We solve system (2) through a standard Newton iteration. Note that, both in the single and multiple shooting cases, the system obtained has more equations than unknown variables. This is due in part to some redundancy in the equations of system (2), that we have kept because it does not add a remarkable computational overhead and provides a certain robustness with respect to convergence. The different number of equations and unknowns is dealt with by implementing minimum-norm, least-squares Newton corrections, using $Q R$ decomposition with column pivoting to solve the corresponding linear systems. More details on this can be found in [13]. In order to differentiate (2) with respect to all the unknowns, we numerically integrate the system of the CRTBP ordinary differential equations together with their first and second variationals. $\oint$ For the numerical integrations, a variable step Runge-KuttaFelbergh method of orders 7 and 8 has been used with a relative tolerance of $10^{-14}$. The absolute tolerances used to stop Newton iterates in the solution of system (2), either in its simple or multiple shooting version, have ranged from $10^{-10}$ to $10^{-12}$.

\section{The Circular Restricted Three-Body Problem}

The CRTBP describes the motion of a particle of infinitesimal mass, moving under the gravitational influence of two massive bodies called primaries, that describe circular orbits around their common centre of mass. We will consider the planar problem, in which the motion of the third body is contained in the plane of motion of the primaries. Taking a (synodic) coordinate system of reference that rotates with the primaries, with the origin located at their centre of mass, and normalised units, we can assume that the primaries have masses $1-\mu$ and $\mu, \mu \in(0,1 / 2]$, their positions are fixed at $(\mu, 0)$ and $(\mu-1,0)$, respectively, and the period of their motions is $2 \pi$. By introducing momenta $p_{x}=\dot{x}-y$ and $p_{y}=\dot{y}+x$, the equations of motion of the CRTBP transform into the

$\S$ An alternative is to use a Taylor integration package for ODE that automatically computes them $[16,1]$. 
following Hamiltonian system

$$
\begin{array}{ll}
\dot{x}=p_{x}+y, & \dot{p}_{x}=p_{y}-\frac{(1-\mu)(x-\mu)}{r_{1}^{3}}-\frac{\mu(x-\mu+1)}{r_{2}^{3}}, \\
\dot{y}=p_{y}-x, & \dot{p}_{y}=-p_{x}-y\left(\frac{1-\mu}{r_{1}^{3}}+\frac{\mu}{r_{2}^{3}}\right)
\end{array}
$$

where $r_{1}=\sqrt{(x-\mu)^{2}+y^{2}}$ and $r_{2}=\sqrt{(x-\mu+1)^{2}+y^{2}}$, and with associated Hamiltonian function

$$
H\left(x, y, p_{x}, p_{y}\right)=\frac{1}{2}\left(p_{x}^{2}+p_{y}^{2}\right)-x p_{y}+y p_{x}-\frac{1-\mu}{r_{1}}-\frac{\mu}{r_{2}} .
$$

Equations (4) satisfy the symmetry

$$
\left(t, x, y, p_{x}, p_{y}\right) \longrightarrow\left(-t, x,-y,-p_{x}, p_{y}\right) .
$$

This implies that, for each solution of Equations (4), if it is not invariant by transformation (5), then this transformation will produce another one, which is seen as symmetric with respect to $y=0$ in configuration space.

The CRTBP has five equilibrium points: the collinear points, $L_{1}, L_{2}$ and $L_{3}$, located on the line containing the primaries, and the equilateral ones, $L_{4}$ and $L_{5}$, both forming an equilateral triangle with the two primaries (see Figure 2). Throughout the paper, we will denote by $h_{i}$ the value of the energy at the equilibrium point $L_{i}, i=1, \ldots, 5$.

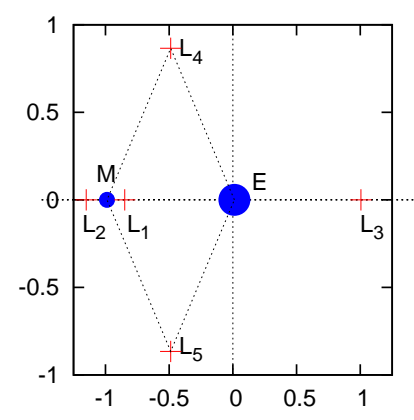

Figure 2. Equilibrium points of the CRTBP for the Earth-Moon problem

The computations in this paper will be related to the dynamics of the CRTBP around the collinear equilibrium points $L_{1}, L_{2}$ and $L_{3}$. We will follow the astrodynamics convention in naming the collinear libration points. That is, if $x_{L_{i}}$ denotes the $x$ coordinate of the $L_{i}$ point, we have $x_{L_{2}} \leq \mu-1 \leq x_{L_{1}} \leq \mu \leq x_{L_{3}}$. Namely, $L_{1}$ is between both primaries, $L_{2}$ is on the left hand side of the small one, and $L_{3}$ is on the right hand side of the large one. See e.g. [23] for more details.

The eigenvalues associated with the collinear equilibrium points are $\{ \pm i \omega, \pm \lambda\}$, so they are centre $\times$ saddle points and Lyapunov's centre theorem (see e.g. [21]) applies. Thus, a one-parametric family of p.o. is born from each collinear equilibrium point, spanning a 2D manifold tangent to real and imaginary parts of the eigenvectors of eigenvalues $\pm i \omega$ at the equilibrium point. These families are known as the Lyapunov 
families of p.o. (LPO). Close to the equilibrium point, they can be parametrised by the energy $h$.

The LPO families inherit hyperbolicity from the collinear libration points, at least for values of $h$ close to $h_{i}$. For these energies, LPO have unstable and stable manifolds. Given two LPO born from different libration points but with the same energy, their unstable and stable manifolds can intersect, giving rise to heteroclinic connections between them. As energy varies, families of heteroclinic orbits are obtained. The next Section presents several numerical continuations of such families, using the methodology introduced in the previous one, according to the applications mentioned in the Introduction.

\section{Results}

In this Section we present the results obtained in the planar CRTBP using the methodology explained previously, considering different scenarios. In performing the continuations of the different families of heteroclinic connections of LPO, the number of multiple shooting points has been increased as needed, except when some trajectory has approached collision. In these cases, we have stopped the continuations. The families could be further continued by regularising the equations of motion (which we have not done).

In looking for families of heteroclinic connections of LPO corresponding to different libration points, we have always followed a similar pattern. More precisely, the steps followed to find families of heteroclinic connections between LPO around $L_{l}$ and LPO around $L_{m}$ are:

(i) For a given $\mu$, consider a value of the energy $h>\max \left(h_{l}, h_{m}\right)$, so there exist planar LPO around both $L_{l}$ and $L_{m}$.

(ii) Consider a LPO around $L_{l}, X_{l}$, and another around $L_{m}, X_{m}$, of that energy level. For the orbit around $L_{l}$ consider the unstable manifold, $W^{u}\left(X_{l}\right)$, and for the one around $L_{m}$ the stable manifold, $W^{s}\left(X_{m}\right)$. In each case, take the branch going towards the other libration point (see, for example, Figure 3 left).

(iii) Consider the $j$-th intersection of the unstable branch and the $k$-th intersection of the stable one with a fixed section $\Sigma$ (it will always be a hyperplane), for fixed $j, k$. We denote them as $W^{u}\left(X_{l}\right) \cap \Sigma^{j}$ and $W^{s}\left(X_{m}\right) \cap \Sigma^{k}$. As the branches of the manifolds are $2 \mathrm{D}$, the intersection with a section is topologically an $S^{1}$ curve except if the manifolds have encountered a collision with the small primary or there exists a heteroclinic connection with less intersections with $\Sigma$.

(iv) Look for the common points of $W^{u}\left(X_{l}\right) \cap \Sigma^{j}$ and $W^{s}\left(X_{m}\right) \cap \Sigma^{k}$. There will be as many families of heteroclinic connections as number of points found.

(v) Apply the continuation procedure (forward and backward in $h$ ) to follow each family. 
Given a heteroclinic connection from a LPO around $L_{l}$ to a LPO around $L_{m}$, the symmetry of Equation (5) will produce a heteroclinic connection in the reverse direction (from a LPO around $L_{m}$ to a LPO around $L_{l}$ ).

In the following subsections we describe several families that have been computed for different values of the mass parameter in the planar CRTBP. In each case, we specify the section $\Sigma$ used to match the invariant manifolds and we include some plots to illustrate the steps described above. The section has always been $\Sigma=\{x=c\}$ for a fixed constant $c$. The curves $W^{u / s} \cap \Sigma^{j / k}$ are represented in either the $\left(y, p_{y}\right)$ or the $\left(p_{x}, p_{y}\right)$ projection of the section $\Sigma$. For each common point of $W^{u / s} \cap \Sigma^{j / k}$ in the projections that has been considered as a connection, the remaining variables have been checked to coincide. We denote by $\left(x_{\Sigma}, \ldots, p_{y_{\Sigma}}\right)$ each common point of the curves on the section belonging to a heteroclinic connection. In all this Section, $X_{i}$ will denote a LPO around $L_{i}$.

\subsection{Heteroclinic connections in the Earth-Moon system}

As a first example, we extend some of the families of heteroclinic connections of LPO computed in [4]. We consider the CRTBP for the Earth-Moon problem with mass parameter $\mu_{E M}=0.012150585$. As $h_{1}<h_{2}$, for values of the energy $h>h_{2}$ there exist LPO around $L_{1}$ and $L_{2}$. Thus, given two planar Lyapunov orbits, $X_{1}, X_{2}$, one around $L_{1}$ and one around $L_{2}$, at the same energy level, we follow a branch of $W^{u}\left(X_{1}\right)$ and a branch of $W^{s}\left(X_{2}\right)$ up to their first intersection with the section $\Sigma=\left\{x=\mu_{E M}-1\right\}$, see Figure 3 left. We plot the curves obtained in the $\left(y, p_{y}\right)$ plane and look for common points (see Figure 3 right). We find two such points, that correspond to two families of heteroclinic connections with no loops around the Moon.
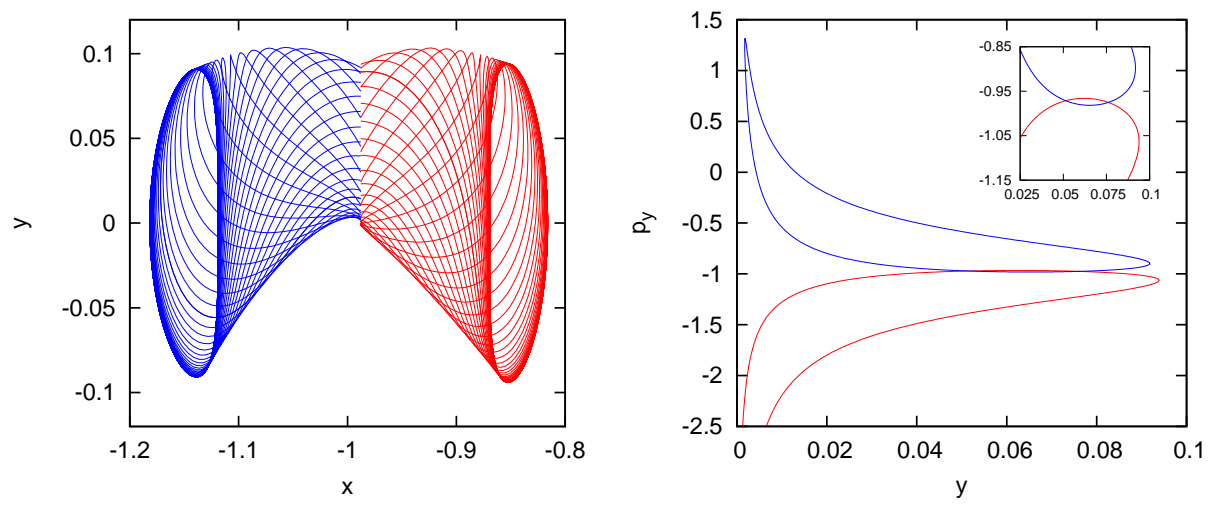

Figure 3. Left: $(x, y)$ projection of the branches of $W^{u}\left(X_{1}\right)$ (red) and $W^{s}\left(X_{2}\right)$ (blue) up to the first crossing with $\Sigma$ for the Earth-Moon CRTBP, for a fixed energy. Right: $\left(y, p_{y}\right)$ projection of the curves $W^{u}\left(X_{1}\right) \cap \Sigma^{1}$ and $W^{s}\left(X_{2}\right) \cap \Sigma^{1}$. The branch $W^{u}\left(X_{1}\right)$ has orbits that collide with the small primary, so the curve $W^{u}\left(X_{1}\right) \cap \Sigma^{1}$ is open and only part of it is shown in the plot.

These two families are considered as one family in [4], labelled as $\mathrm{He}_{1,2}^{0}$, for values 
of the energy up to approximately $h \simeq-1.5685$. Without regularisation, we are able to follow the same family up to values of the energy of -1.512772726 . See Figure 4, left, where the characteristic curves of the two families are shown in the $\left(h, y_{\Sigma}\right)$ plane.
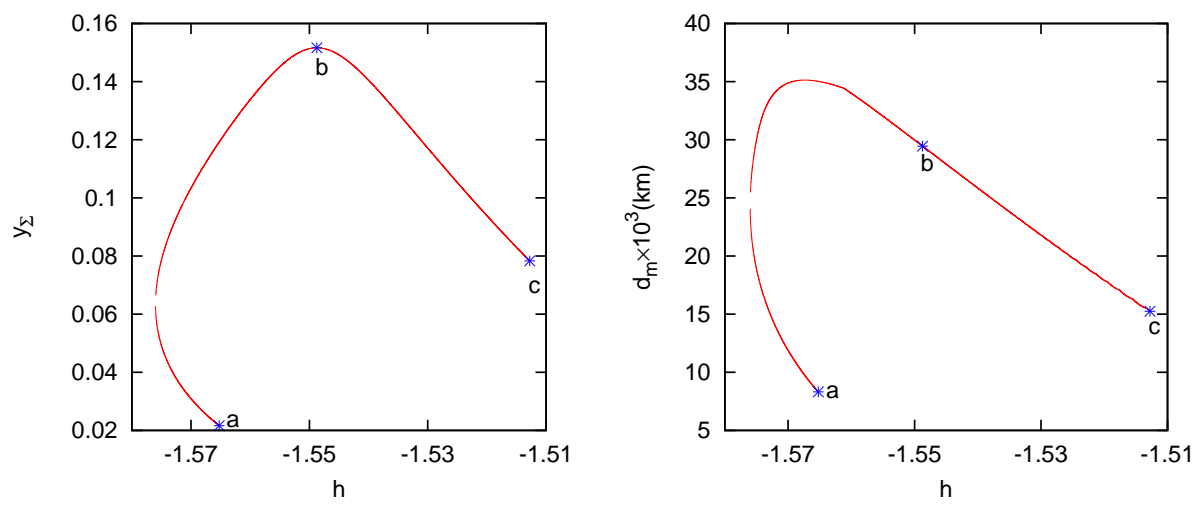

Figure 4. Characteristic curves of two families of heteroclinic connections of the Earth-Moon CRTBP. Left: the curves in the $\left(h, y_{\Sigma}\right)$ plane. Right: Minimum distance to the Moon in $\mathrm{km}$ of each heteroclinic connection (average distance from the Earth to the Moon: $384400 \mathrm{~km}$ )

We have also computed the minimum distance $d_{m}$ from each heteroclinic connection to the Moon. Along one of the families, $d_{m}$ decreases from the beginning of the family, see Figure 4 right. We have stopped the computations when that distance reaches a value of approximately 0.02 (about $6300 \mathrm{~km}$ from the surface of the Moon). In Figure 5, left, the orbit with the minimum $d_{m}$ computed is shown. Along the other family, the minimum distance $d_{m}$ increases initially, and after reaching a maximum value of 0.0766 (about $27700 \mathrm{~km}$ from the surface of the Moon), it decreases. This is mainly due to the fact that the width of the Lyapunov orbits involved increases (see Figure 5, right).

In Figure 5, the heteroclinic orbits labelled with letters a, b, and c in Figure 4 are shown. Some data corresponding to these orbits can be found in Table 1.

(a)

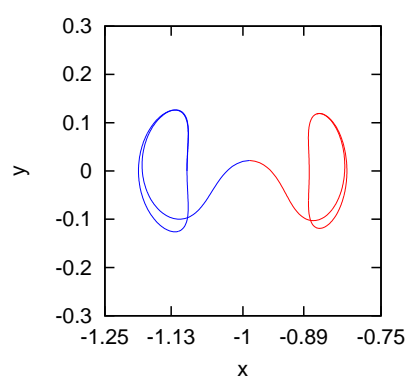

(b)

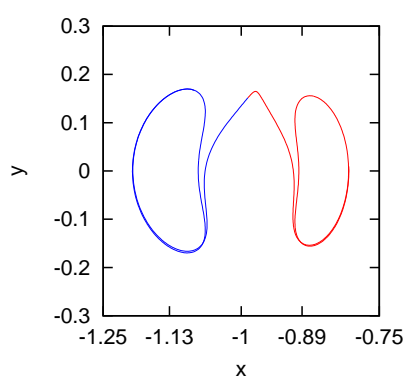

(c)

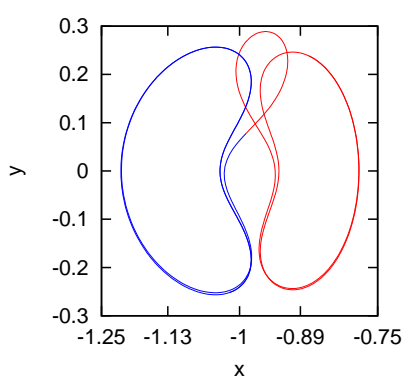

Figure 5. Heteroclinic connections between LPO around $L_{1}$ and $L_{2}$ of the CRTBP for the the Earth-Moon value of the mass parameter. From left to right, the orbits correspond to the points labelled with letters a, b, c in Figure 4. The red and blue colours correspond to the trajectory on the unstable and stable manifold respectively. 


\begin{tabular}{|c|c|l|c|}
\hline & $h$ & \multicolumn{1}{|c|}{$y_{\Sigma}$} & \multicolumn{1}{c|}{$d_{m}$} \\
\hline a & -1.565229525561280 & 0.02162260888134571 & 0.02162260624628988 \\
\hline b & -1.548737225565584 & 0.1516356943492464 & 0.07661260101642152 \\
\hline c & 1.512772725566362 & 0.07830352581009974 & 0.03965928526119706 \\
\hline
\end{tabular}

Table 1. Values of energy $h, y_{\Sigma}$ and $d_{m}$ of the orbits shown in Figure 5

\subsection{Homoclinic-heteroclinic connections and resonance transitions in the Sun-Jupiter system}

We consider the Sun-Jupiter CRTBP, with mass parameter $\mu_{S J}=0.000953875$, and a range of values of the energy $h>h_{2}$, so that the Hill region (the region in the configuration $(x, y)$ plane where the motion is allowed) has just one component, but three different zones (see Figure 6): an interior region around the Sun, an exterior region surrounding both primaries up to infinity, and a two-bottleneck region around Jupiter. The interior and exterior regions are connected via the region around Jupiter where the Lyapunov orbits of that specific energy level exist. The transitions between these three regions are governed by the invariant manifolds structures associated with the p.o. around $L_{1}$ and $L_{2}$, as well as the existence of heteroclinic and homoclinic connections, and transit and non-transit orbits. Transit and non-transit orbits were introduced in [5], and can be compactly defined as follows: a trajectory approaching a LPO, either forward or backward in time from one of the three regions described above, is considered transit if it traverses the bottleneck corresponding to the LPO and goes to the next region. On the contrary, it is considered non-transit if it bounces back to the region it comes from. Transit orbits are known to lie in the interior of the invariant manifold tubes of LPO, that separate them from non-transit orbits $[5,20,18]$. In addition to this, we will denote by inner orbits (or trajectories) those that stay around the Sun in the interior of the Hill's region, and outer orbits those that surround the Sun and Jupiter in the exterior region.

In [18], the authors show how homoclinic orbits of LPO around $L_{1}$ and $L_{2}$, together with a heteroclinic connection between them for the same value of the energy, can be combined in a dynamical chain according to a sequence of regions to visit (exterior, Jupiter, interior), called itinerary. This dynamical chain is the backbone of the collection of orbits -denoted as a dynamical channel- that follow the same itinerary. For the specific value of the energy $h=-1.51452351743876$ (Jacobi constant $C_{J}=3.03$ ), they actually compute the corresponding homoclinic and heteroclinic connections and an orbit that makes the transition from the exterior region to interior region, and viceversa, through the Jupiter region, in such a way that it follows a 2:3 resonance with Jupiter in the exterior region, and a 3:2 resonance in the interior one. This orbit is provided as a dynamical explanation of the resonance transitions followed by comet Oterma.

Our goal in this Section is to follow families of heteroclinic connections between 

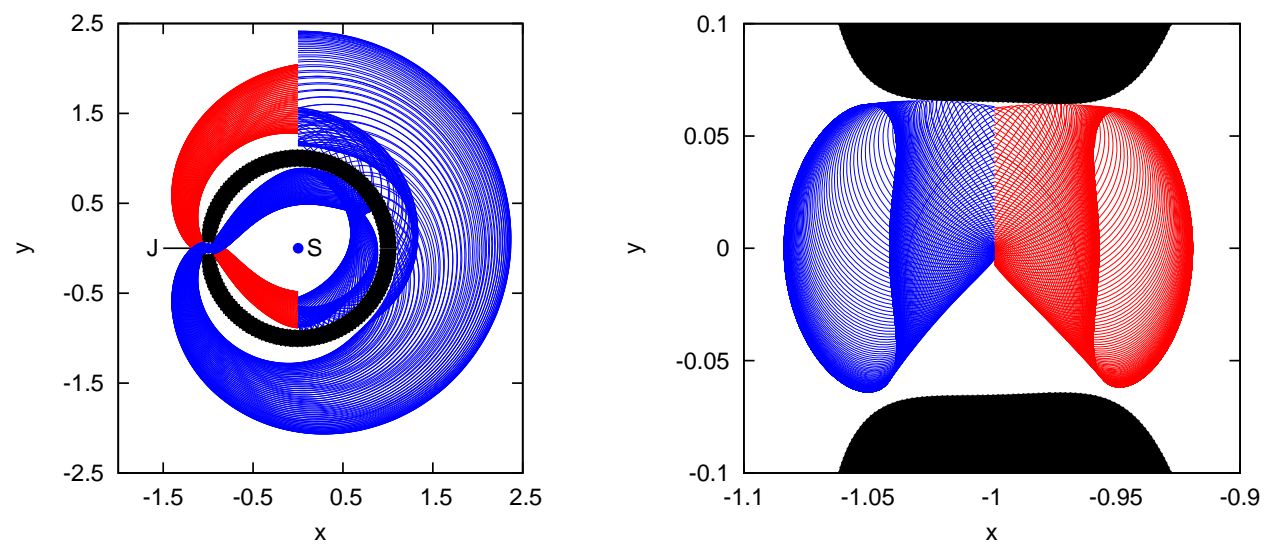

Figure 6. Projection in the $(x, y)$ plane of some branches of the invariant manifolds of LPO around $L_{1}$ and $L_{2}$ of the Sun-Jupiter CRTBP, for $h=-1.5127313471774606$. The forbidden region for motion is also represented (in black). Left: Inner branches of $W^{u / s}\left(X_{1}\right)$ and outer branches of $W^{u / s}\left(X_{2}\right)$. Right: Detail of the region around Jupiter, a branch of $W^{u}\left(X_{1}\right)$ and a branch of $W^{s}\left(X_{2}\right)$. Orbits in the unstable manifolds are plotted in red, and orbits the stable ones in blue.

LPO around $L_{1,2}$ and families of homoclinic connections of LPO around $L_{1,2}$, in order to find the ranges of energy in which they exist simultaneously, making dynamical chains possible. For each type of connection, we will follow several families, that provide several possibilities for a dynamical chain corresponding to a given itinerary. In the case of homoclinic connections, different families will be found to correspond to different resonances.

The different resonances will be identified in terms of two-body dynamics. A Keplerian orbit around Jupiter (a solution of the two-body problem), is said to be in a $p: q$ resonance when its mean motion is $p / q$, where $p$ is the number of revolutions of the massless particle around the Sun, and $q$ the number of revolutions of Jupiter (see, e.g. [23]). Thanks to the use of normalised units, in the inertial frame the mean motion resonance of the orbit with respect to Jupiter is $a^{-3 / 2}$, where $a$ is the semimajor axis computed as a two-body orbital element. In terms of synodic coordinates, $a$ can be written as

$$
a^{-1}=2 / r-v^{2},
$$

being $r^{2}=x^{2}+y^{2}, v^{2}=p_{x}{ }^{2}+p_{y}{ }^{2}$. For solutions of the two-body problem, $a$ remains constant. For trajectories of the CRTBP it is not constant, but it will be approximately constant for trajectories that behave essentially as a two-body solution. This happens for the inner and outer homoclinic connections providing the dynamical chains studied in [18]. As a value of reference for each such homoclinic connection, we will compute the value of $a$ at the point of the intersection of the two manifolds. We will see how $a^{-3 / 2}$ varies along a family of homoclinic connections and how it is related to resonances.

In the remaining of this Subsection, we first compute and describe families of heteroclinic connections between LPO around $L_{1}$ and $L_{2}$. After that, we compute and 
describe families of homoclinic connections of LPO around $L_{1}$ and of LPO around $L_{2}$. We relate different families to different resonances according to the previous discussion. We end this Subsection with a comment on the representation of resonant transit orbits.

Homoclinic connections are computed using the methodology of [3], of which, as mentioned in the introduction, the methodology of this paper is an extension.

4.2.1. Heteroclinic connections in the Sun-Jupiter system. We start looking for heteroclinic connections from LPO around $L_{1}$ to LPO around $L_{2}$ in the Sun-Jupiter system. We compute two kinds of heteroclinic orbits: those with no loops around Jupiter, and those with just one loop around the planet. Concerning the latter, for example, in Figure 7 left, the invariant manifolds up to the second intersection with the section $\Sigma=\left\{x=\mu_{S J}-1\right\}$ are plotted in configuration space, for the value of the energy $h=-1.5180226$. In Figure 7 right, the curves $W^{u}\left(X_{1}\right) \cap \Sigma^{2}$ and $W^{s}\left(X_{2}\right) \cap \Sigma^{2}$, for the same energy level, are plotted. The two common points of the curves belong to two families of heteroclinic connections with a loop around Jupiter. We follow these families using the method of Section 2.
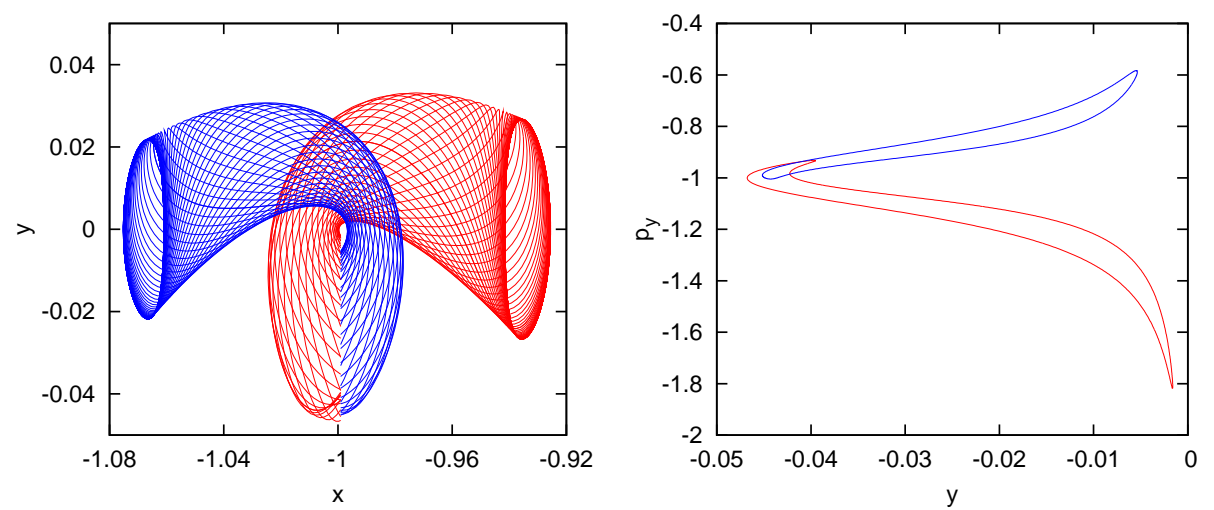

Figure 7. Left: $(x, y)$ projection of the branches of $W^{u}\left(X_{1}\right)$ (red) and $W^{s}\left(X_{2}\right)$ (blue) up to the second crossing with $\Sigma$ for the Sun-Jupiter CRTBP, and a certain value of the energy. Right: $\left(y, p_{y}\right)$ projection of the curves $W^{u}\left(X_{1}\right) \cap \Sigma^{2}$ and $W^{s}\left(X_{2}\right) \cap \Sigma^{2}$.

The families are denoted by $\mathrm{He}_{j}^{k}$, where $k$ denotes the number of loops and $j=1,2, \ldots$ is the index used to label each family. In Figure 8 left, the characteristic curves of the aforementioned families of each kind are shown in the $\left(h, y_{\Sigma}\right)$ plane. We also compute the minimum distance to Jupiter of each orbit, see Figure 8 right. As we see, two of the families tend quickly to the small primary, so regularisation would be required in order to further follow the corresponding branches. In both cases (orbits with no loops, or with one loop around Jupiter) two of the families can be computed up to values of the energy about -1.507 .

In Table 2, some data of the selected orbits marked in Figure 8 is given. These orbits are shown in Figure 9. 

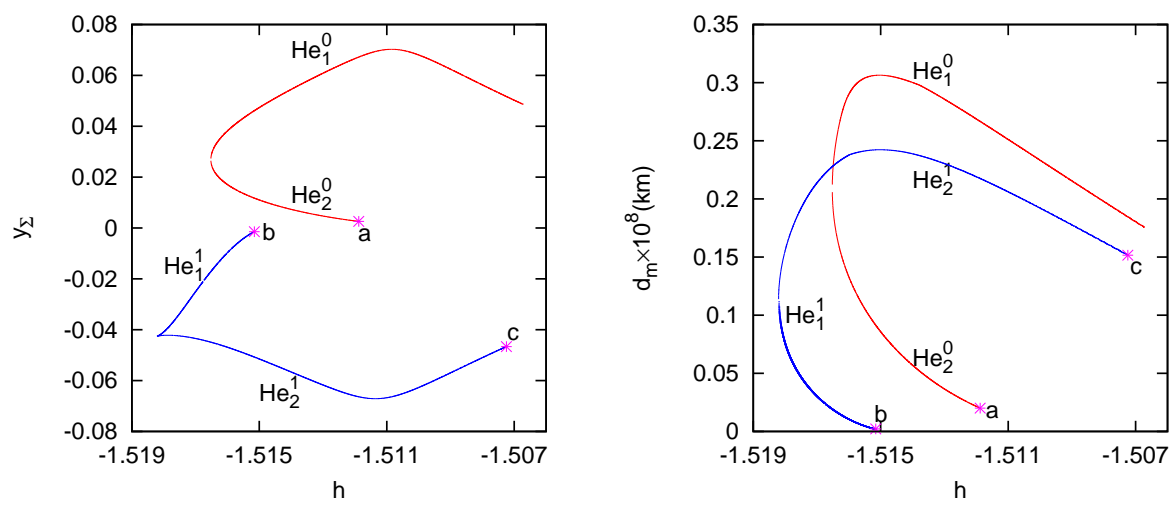

Figure 8. Left: two families of heteroclinic connections with no loops around Jupiter $\left(\mathrm{He}_{j}^{0}, j=1,2\right)$, and two families with one loop around Jupiter $\left(\mathrm{He}_{j}^{1}, j=1,2\right)$. Right: Minimum distance to Jupiter in $\mathrm{km}$ (semimajor axis of Jupiter: $7.7834082 \times 10^{8} \mathrm{~km}$ ).

\begin{tabular}{|c|c|c|c|}
\hline & $h$ & $y_{\Sigma}$ & $d_{m}$ \\
\hline $\mathrm{a}$ & -1.511880607561459 & $0.258030744057995 \times 10^{-2}$ & $0.258030744058029 \times 10^{-2}$ \\
\hline $\mathrm{b}$ & -1.515156607561478 & $-0.145692618451761 \times 10^{-2}$ & $0.271786667180622 \times 10^{-3}$ \\
\hline $\mathrm{c}$ & -1.507246907557488 & -0.046616960243633 & 0.019477513097560 \\
\hline
\end{tabular}

Table 2. Values of energy $h, y_{\Sigma}$ and $d_{m}$ for the selected orbits marked in Figure 8.

(a)

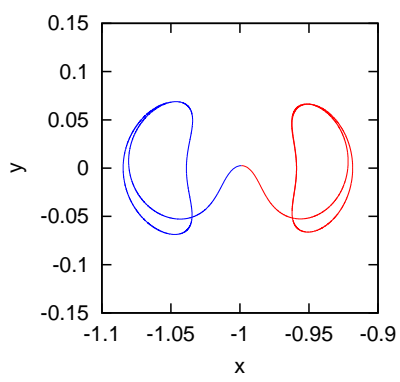

(b)

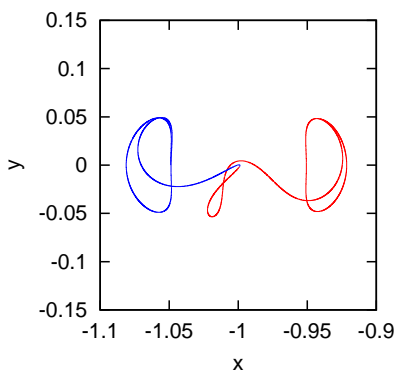

(c)

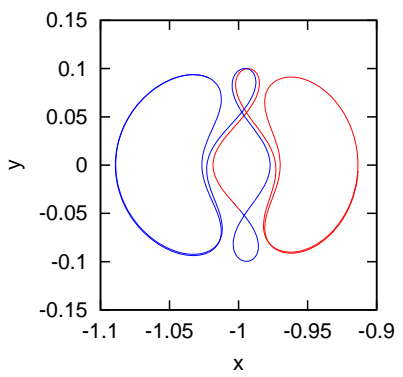

Figure 9. Examples of heteroclinic connections between LPO around $L_{1}$ and $L_{2}$ for the Sun-Jupiter value of the mass parameter. The red and blue colours correspond to the trajectory on the unstable and stable manifold respectively.

4.2.2. Homoclinic connections in the Sun-Jupiter system. In this Section we are interested in homoclinic connections to LPO around $L_{1}$ (in the interior region) and around $L_{2}$ (in the exterior one), and the behaviour of the keplerian semimajor axis of these homoclinics.

We start with the inner homoclinics, that is, homoclinic connections of LPO around $L_{1}$ that revolve around the Sun. We always use the section $\Sigma=\{x=0\}$ and consider orbits that revolve once around the Sun, so we look for the first intersection of $W^{u}\left(X_{1}\right)$ and the second intersection of $W^{s}\left(X_{1}\right)$ with $\Sigma$ (see Figure 6 left). In Figure 10, we show these curves, in the $\left(p_{x}, p_{y}\right)$ plane, for three different values of the energy. The shadowed 
regions correspond to transit orbits forward and backward in time.
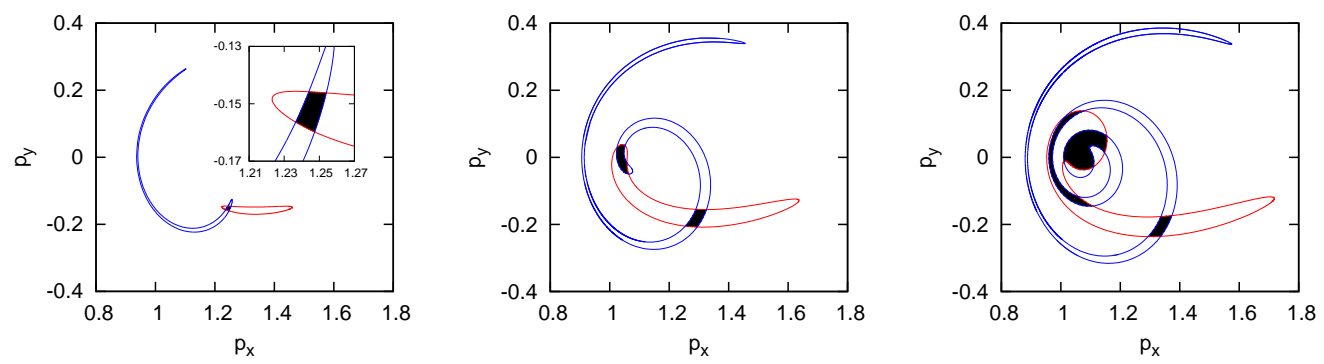

Figure 10. $\left(p_{x}, p_{y}\right)$ projection of the curves $W^{u}\left(X_{1}\right) \cap \Sigma^{1}$ and $W^{s}\left(X_{1}\right) \cap$ $\Sigma^{2}$ for the energy values, from left to right, $h=-1.5185311413912728, h=$ $-1.5125206068732759, h=-1.5063309905638478$.

Each intersection point between both curves belongs to a homoclinic orbit. We have followed the twelve corresponding families of inner homoclinics, $\mathrm{Hi}_{j}, j=1, \ldots, 12$ of the Sun-Jupiter CRTBP problem. In Figure 11 left, the characteristic curves of the families in the $\left(h, y_{\Sigma}\right)$ plane are shown. Although, as previously said, we stop the continuations when approaching a collision (in this case, a close passage to Jupiter of the Lyapunov orbits), we are able to obtain homoclinics for a range of values of the energy larger than the range of the heteroclinic connections computed. In the same Figure, right, we plot, for each family, the energy and the value of the mean motion of the approximated ellipse, $a^{-3 / 2}$. We can see that the families are organised in groups of four around different resonances, 3:2, 4:3 and 5:4. At each group, two of the families are of symmetric orbits, whereas the other two are of non-symmetric. For the families computed, the non-symmetric ones correspond always to the families with the value of $a^{-3 / 2}$ closer to the resonance, see Figures 11 right and 15 right.

Next, we proceed with the homoclinic connections to LPO around $L_{2}$ that revolve once around both the Sun and Jupiter, that is, outer homoclinics. Again we use the section $\Sigma=\{x=0\}$ and look for the first intersection of $W^{u}$ and the second intersection of $W^{s}$ with $\Sigma$ (see Figure 6, left). Similarly as before, we look for the intersections of the curves $W^{u}\left(X_{2}\right) \cap \Sigma^{1}$ and $W^{s}\left(X_{2}\right) \cap \Sigma^{2}$. In Figure 12, we show these curves in the $\left(p_{x}, p_{y}\right)$ plane for three different values of the energy. Again, we follow twelve families of homoclinic connections, $\mathrm{Ho}_{j}, j=1, \ldots, 12$ that correspond to the twelve intersections of both curves.

In Figure 13, left, we plot the characteristic curves, in the $\left(h, y_{\Sigma}\right)$ plane, of the families computed. The range of energy for which we compute outer homoclinics is essentially the same as for the inner ones. We also compute the value of the semimajor axis and, in Figure 13, right, the characteristic curves in the $\left(h, a^{-3 / 2}\right)$ plane are shown. We can observe that, as the families of inner homoclinics, they are organised in groups of four. In this case, around the resonances 1:2, 2:3 and 3:4.

In Figure 14 some inner and outer homoclinic orbits of different families are shown. 

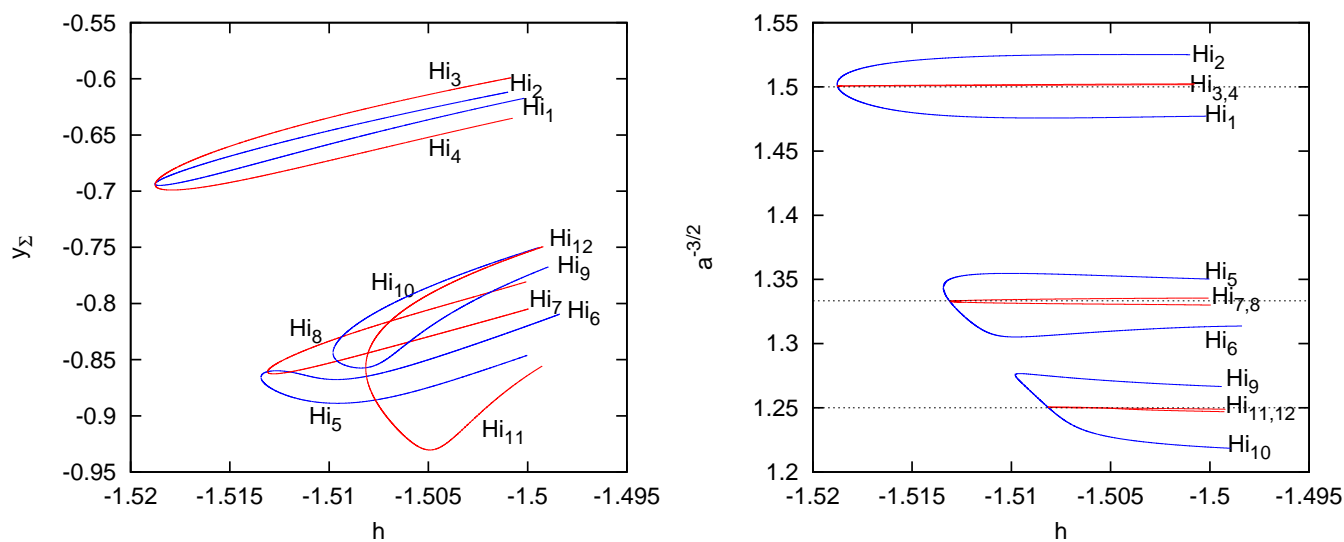

Figure 11. Different families of homoclinic connections to LPO around $L_{1}$ of the SunJupiter CRTBP that surround once the Sun. In blue, families of symmetric orbits; in red, families of non-symmetric orbits. Left: characteristic curves in the $\left(h, y_{\Sigma}\right)$ plane. Right: families in the $\left(h, a^{-3 / 2}\right)$ plane. The dotted lines correspond to the $3: 2,4: 3$ and $5: 4$ resonances.
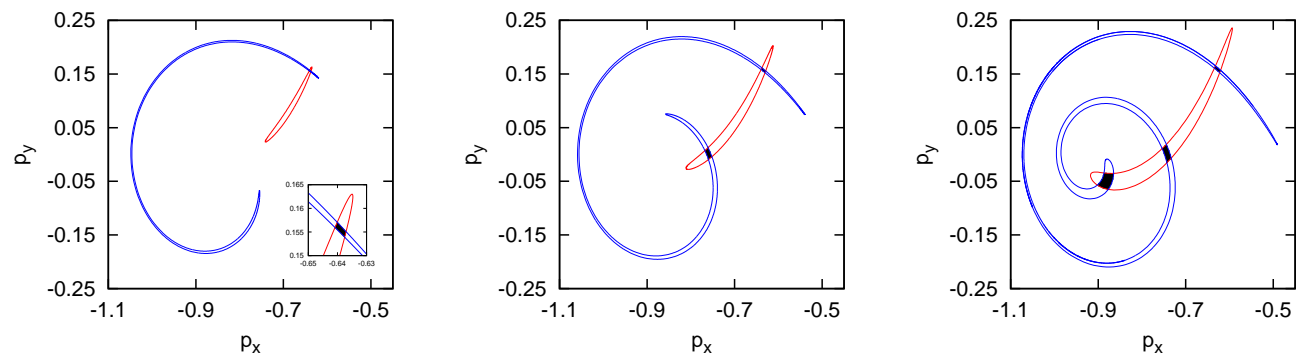

Figure 12. $\left(p_{x}, p_{y}\right)$ projection of the curves $W^{u}\left(X_{2}\right) \cap \Sigma^{1}$ and $W^{s}\left(X_{2}\right) \cap$ $\Sigma^{2}$ for the energy values, from left to right, $h=-1.5173634301253143, h=$ $-1.5147777489155008, h=-1.5109240998869069$.

In Table 3 the minimum (starting) value for which each couple of families exist is given, as well as the resonance that corresponds to each family.

\begin{tabular}{|c|l|c||c|l|c|}
\hline Resonance & $\begin{array}{l}\text { Inner } \\
\text { family }\end{array}$ & \multicolumn{1}{|c||}{$h$} & Resonance & $\begin{array}{l}\text { Outer } \\
\text { family }\end{array}$ & \multicolumn{1}{c|}{$h$} \\
\hline \multirow{2}{*}{$3: 2$} & $\mathrm{Hi}_{1,2}$ & -1.5187738680438660 & $1: 2$ & $\mathrm{Ho}_{1,2}$ & -1.5176875927559890 \\
& $\mathrm{Hi}_{3,4}$ & -1.5187644031439360 & & $\mathrm{Hi}_{3,4}$ & -1.5176861758563389 \\
\hline \multirow{2}{*}{$4: 3$} & $\mathrm{Hi}_{5,6}$ & -1.5134324575378211 & $2: 3$ & $\mathrm{Ho}_{5,6}$ & -1.5164193318780410 \\
& $\mathrm{Hi}_{7,8}$ & -1.5130995908322500 & & $\mathrm{Ho}_{7,8}$ & -1.5164069483330780 \\
\hline \multirow{2}{*}{$5: 4$} & $\mathrm{Hi}_{9,10}$ & -1.5098028285856659 & $3: 4$ & $\mathrm{Hi}_{9,10}$ & -1.5119690998868209 \\
& $\mathrm{Hi}_{11,12}$ & -1.5081547305639951 & & $\mathrm{Ho}_{11,12}$ & -1.5118090998870310 \\
\hline
\end{tabular}

Table 3. Minimum value of the energy $h$, for which families of inner and outer homoclinic connections exist.

Figure 15 provides an example of a compact representation of all the inner transit 

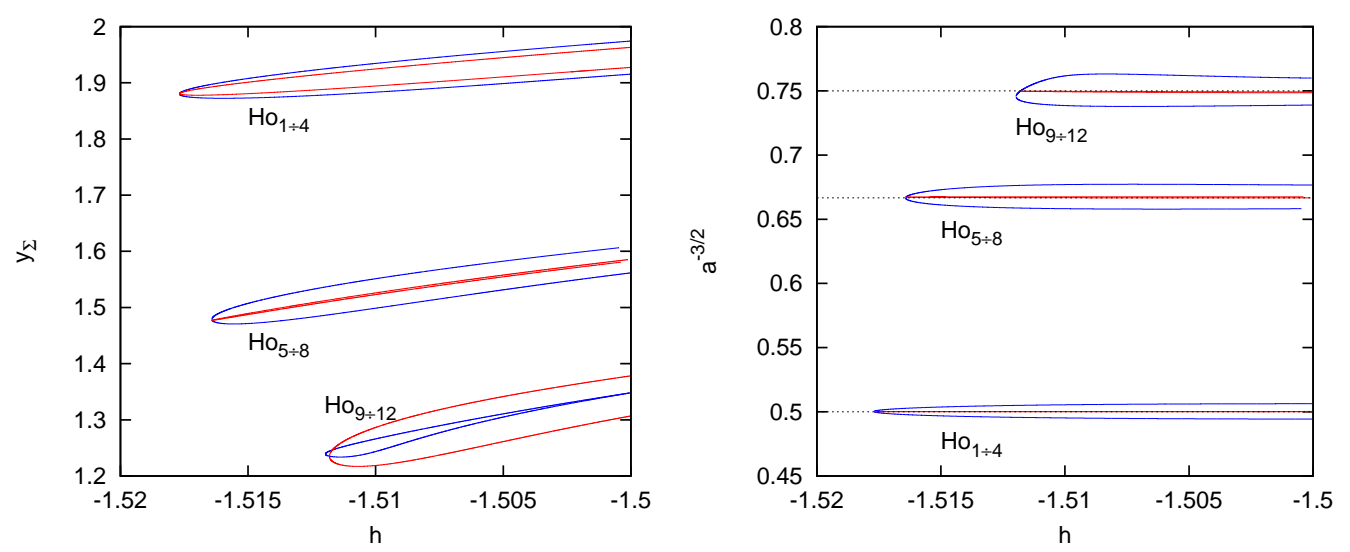

Figure 13. Different families of outer homoclinic connections to LPO around $L_{2}$ of the Sun-Jupiter CRTBP. In blue, families of symmetric orbits; in red, families of nonsymmetric orbits. Left: characteristic curves in the $\left(h, y_{\Sigma}\right)$ plane. Right: families in the $\left(h, a^{-3 / 2}\right)$ plane. The dotted lines correspond to the $1: 2,2: 3$ and 3:4 resonances.
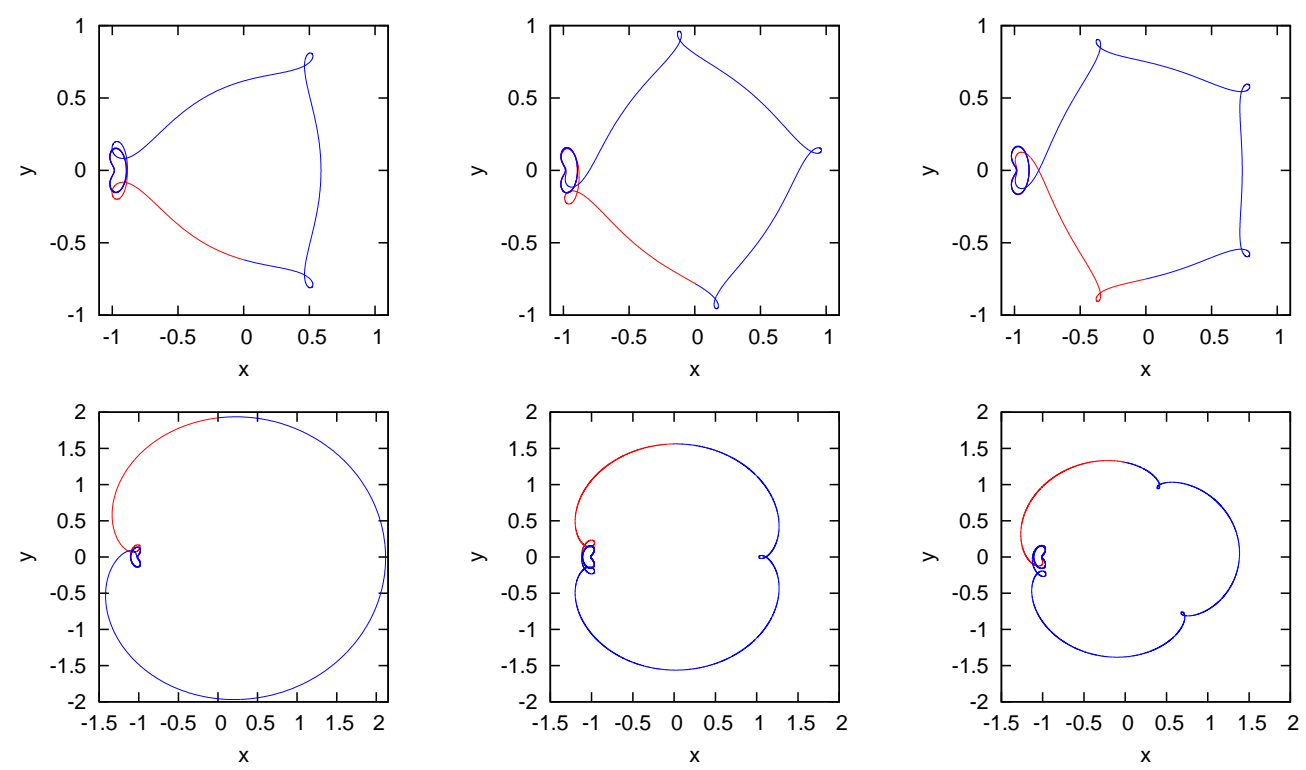

Figure 14. Examples of inner (top) and outer (bottom) homoclinic connections (symmetric and non-symmetric) of LPO around $L_{1}$ and $L_{2}$ for the Sun-Jupiter value of the mass parameter. The orbits correspond to the highest value of $h$ computed from the families inner families $\mathrm{Hi}_{1}, \mathrm{Hi}_{8}$ and $\mathrm{Hi}_{10}$ and from the outer families $\mathrm{Ho}_{3}, \mathrm{Ho}_{7}$ and $\mathrm{Ho}_{10}$ (from left to right).

The red and blue colours correspond to the trajectory on the unstable and stable manifold respectively.

trajectories of an energy level associated to a resonance (similar plots can be done for outer transit trajectories). The left plot is actually a zoom of Figure 10 left. In it, a region of transit orbits has as boundary the segments of the $W^{s}\left(X_{1}\right) \cap \Sigma^{1}$ and $W^{u}\left(X_{1}\right) \cap \Sigma^{2}$ curves delimited by the four homoclinic connections $\mathrm{Hi}_{1,2,3,4}$. The right 
plot of Figure 15 displays the same curves in the $\left(p_{x}, a^{-3 / 2}\right)$ plane, showing that all the transit trajectories of the region are associated to the 3:2 resonance.

It must be noted that, since for each connection belonging to $\mathrm{Hi}_{i}$ we have the values of all the variables of Equation (3) along the family, performing the continuations of the $\mathrm{Hi}_{1,2,3,4}$ families provides all the necessary information to generate the borders of the corresponding region of transit orbits at each energy level. For example, the segment of $W^{s}\left(X_{1}\right) \cap \Sigma^{2}$ between $\mathrm{Hi}_{1}$ and $\mathrm{Hi}_{4}$ is delimited by the $\theta^{s}$ values corresponding to these two connections. The same observation applies to the section plots of all the families of homoclinic and heteroclinic connections we have followed. In this way, the computations presented in this Subsection can be viewed as a first step towards the automatic generation of trajectories in the dynamical channel associated to a given itinerary.
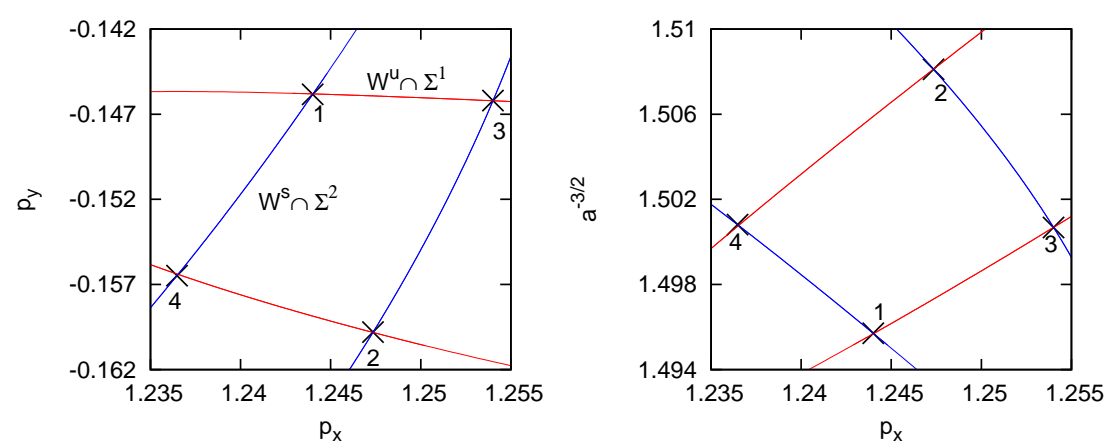

Figure 15. Curves $W^{u} \cap \Sigma^{1}$ (red) and $W^{s} \cap \Sigma^{2}$ (blue) for the value $h=$ -1.51853114139 . Left: detail of the curves in the $\left(y, p_{y}\right)$ plane around the intersection points corresponding to the families $\mathrm{Hi}_{j}, j=1, \ldots, 4$. The numbers correspond to the family that each point belongs to. Right: the same curves in the $\left(p_{x}, a^{-3 / 2}\right)$ plane.

\subsection{Heteroclinic connections between $L P O$ around $L_{3}$ and $L_{2}$}

As a final example of application of the procedure presented for the continuation of heteroclinic connections of p.o., in this Subsection we will compute two families of heteroclinic connections of LPO around $L_{2}$ and $L_{3}$. As far as we know, no heteroclinic connection has been computed involving a LPO around $L_{3}$ and LPO around another collinear libration point. The use of purely numerical procedures is mandatory in this case, since semianalytical procedures do not produce useful approximations in the neighbourhood of $L_{3}[15]$.

We will first look for a value of $\mu$ for which there is a heteroclinic connection between $L_{3}$ and a LPO around $L_{2}$. For that, we fix a Poincaré section $\Sigma=\{x=0\}$, and for any given $\mu$, we perform the following steps:

- We compute $L_{3}$, its energy value $h_{3}$ and the LPO around $L_{2}$ for that energy level. We consider the branch of the unstable manifold $W^{u}\left(L_{3}\right)$ that goes to the $y<0$ 
region, and we follow it up to the first crossing with $\Sigma$ (see Figure 16, left). So we obtain a point $P_{L_{3}, \Sigma}^{\mu}=\left(x_{\Sigma}, \ldots, p_{y_{\Sigma}}\right)$.
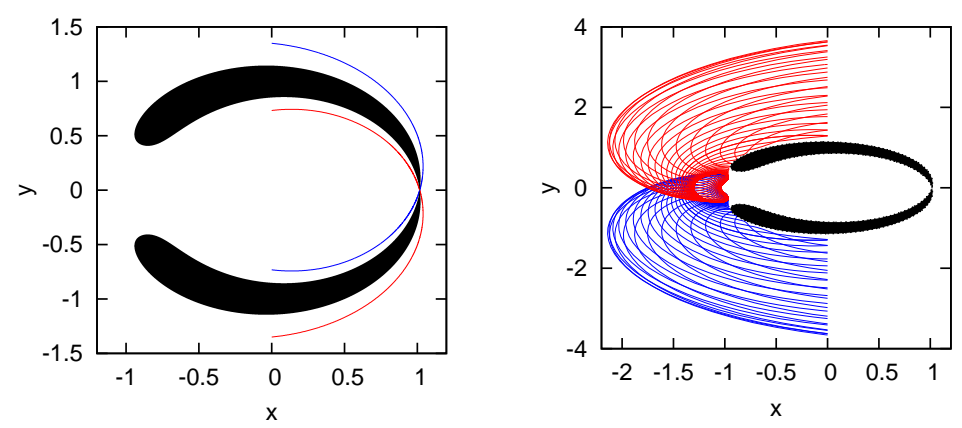

Figure 16. For $\mu=0.04$, projection in $(x, y)$ of the invariant manifolds (unstable in red, stable in blue) of the equilibrium point $L_{3}$ (left, all branches of each invariant manifolds are plotted), and of the LPO around $L_{2}$ with the same energy (right, only the outer branches), up to the section $\Sigma=\{x=0\}$. The region in black corresponds to the forbidden region of motion.

- Similarly, we follow the outer branch of $W^{s}\left(X_{2}\right)$ up to $\Sigma$ (see Figure 16, right), and compute the distance between the point $P_{L_{3}, \Sigma}^{\mu}$ and the set $W^{s}\left(X_{2}\right) \cap \Sigma^{1}$.

Varying $\mu$, we obtain the function $d_{\min }(\mu)$. This function is plotted in Figure 17, left, where we can see that it becomes zero at a single value of $\mu$, which is $\mu_{h}=$ 0.04005993174156. Therefore, for this value of $\mu$ there exists a heteroclinic connection between the equilibrium point $L_{3}$ and a LPO around $L_{2}$ (see Figure 17, right). The
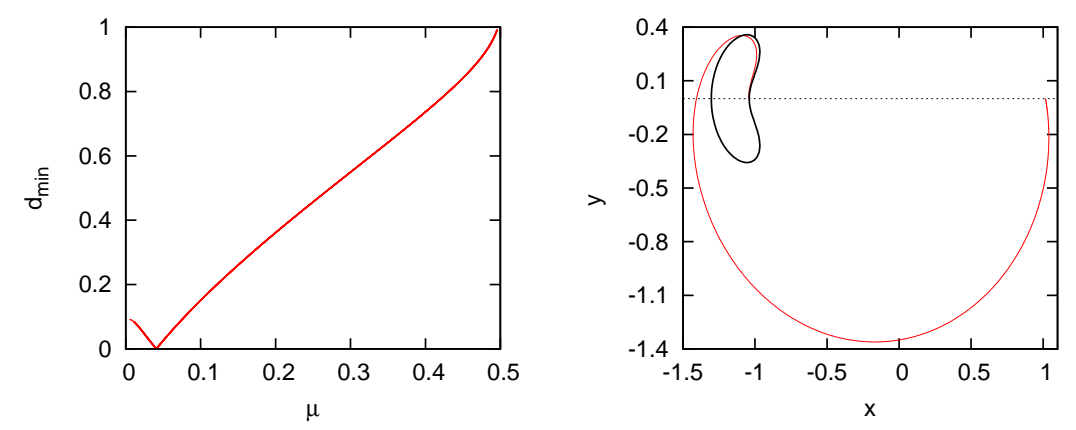

Figure 17. Left: Function $d_{\min }(\mu)$ (see the text for details). Right: A heteroclinic orbit between $L_{3}$ and a LPO around $L_{2}$ (in black) for $\mu=\mu_{h}$.

application of the symmetry of Equation (5) will produce an heteroclinic connection between the Lyapunov orbit and $L_{3}$ in the opposite direction, that will follow the branch of the stable manifold of $W^{s}\left(L_{3}\right)$ that goes to $y>0$ and the outer unstable manifold $W^{u}\left(X_{2}\right)$.

For the value $\mu=\mu_{h}$, increasing the value of the energy there appear LPO around $L_{3}$ with heteroclinic connections to LPO around $L_{2}$, and the same will happen for values of $\mu$ close to $\mu_{h}$ for certain values of the energy. We fix $\mu=0.04$, and follow the same 
strategy as in previous cases. We find two families of heteroclinic connections, whose characteristic curves are plotted in Figure 18.

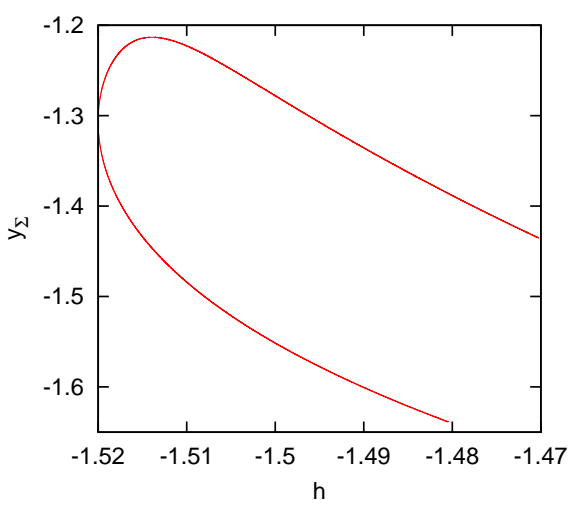

Figure 18. Two families of heteroclinic connections between LPO around $L_{3}$ and $L_{2}$.

The same procedure, but taking into account the $k$-th crossing with the section $\Sigma$ for $k>2$, will provide heteroclinic connections with $k-1$ loops around the large primary. In any case, it is always necessary a study of the suitable values of $\mu$ to look for heteroclinic connections between LPO around $L_{3}$ and $L_{2}$. Furthermore, considering the branch of $W^{u}\left(X_{3}\right)$ that goes to $y>0$, and the inner stable branch of $W^{s}\left(X_{1}\right)$, heteroclinics between LPO around $L_{3}$ and $L_{1}$ will be obtained.

\section{Conclusions}

We have presented a numerical method to carry out the continuation of families of heteroclinic connections between hyperbolic periodic orbits. Compared with previous methodology, our approach presents two advantages: it overcomes the convergence restrictions of semianalytical techniques and provides the automation of the matching process of manifolds of periodic orbits in a section when varying a parameter for the continuation of a family.

We have applied our methodology to the CRTBP, for different values of the mass parameter. In particular we have compared our results with existing ones, and we have also obtained new families. In the case of the resonance transitions in the Sun-Jupiter system studied in [18], we have determined ranges of energy in which they are possible, as well as enlarged the choice of resonances that can be connected.

\section{Acknowledgements}

E. Barrabés and J.M. Mondelo are partially supported by the MCyT/FEDER grants MTM2006-05849/Consolider and MTM2010-16425. J.M. Mondelo is also supported by the MCyT/FEDER grant MTM2011-26995-C02-01 and the Catalan grant 2009SGR410. M. Ollé is partially supported by the MCyT/FEDER grants MTM2009-06973, MTM2012-31714 and 2009SGR-859. 


\section{References}

[1] A. Abad, R. Barrio, F. Blesa, and M. Rodriguez. Algorithm 924: TIDES, a Taylor series integrator for differential equations. ACM Transactions on Mathematical Software, 39(1), 2012.

[2] E.L. Allgower and K. Georg. Numerical continuation methods. An Introduction., volume 13 of Springer Series in Computational Mathematics. Springer-Verlag, Berlin, 1990.

[3] E. Barrabés, J. M. Mondelo, and M. Ollé. Numerical continuation of families of homoclinic connections of periodic orbits in the RTBP. Nonlinearity, 22(12):2901-2918, 2009.

[4] E. Canalias and J. J. Masdemont. Homoclinic and heteroclinic transfer trajectories between planar Lyapunov orbits in the sun-earth and earth-moon systems. Discrete Contin. Dyn. Syst., $14(2): 261-279,2006$.

[5] C.C Conley. Low energy transit orbits in the restricted three-body problem. SIAM Journal on Applied Mathematics, 16(4):732-746, 1968.

[6] A. Delshams, J. Masdemon, and P. Roldan. Computing the scattering map in the spatial Hill's problem. Discrete and Continuous Dynamical Systems, series B, 10(2, 3), 2008.

[7] G. Gómez, A. Jorba, J. Masdemont, and C. Simó. Dynamics and Mission Design Near Libration Point Orbits - Volume 3: Advanced Methods for Collinear Points. World Scientific Publishing Co. Inc., 2001.

[8] G. Gómez, A. Jorba, J. Masdemont, and C. Simó. Dynamics and Mission Design Near Libration Point Orbits - Volume 4: Advanced Methods for Triangular Points. World Scientific, 2001. Reprint of ESA Report Study of Poincaré Maps for Orbits Near Lagrangian Points, 1993.

[9] G. Gómez, W. S. Koon, M. W. Lo, J. E. Marsden, J. Masdemont, and S. D. Ross. Connecting orbits and invariant manifolds in the spatial Restricted Three-Body Problem. Nonlinearity, 17(5):1571-1606, 2004.

[10] G. Gómez, J. Llibre, R. Martínez, and C. Simó. Dynamics and Mission Design Near Libration Point Orbits - Volume 1: Fundamentals: The Case of Collinear Libration Points. World Scientific Publishing Co. Inc., 2001.

[11] G. Gómez, J. Llibre, R. Martínez, and C. Simó. Dynamics and Mission Design Near Libration Point Orbits - Volume 2: Fundamentals: The Case of Triangular Libration Points. World Scientific Publishing Co. Inc., 2001.

[12] G. Gómez, M. Marcote, and J. M. Mondelo. The invariant manifold structure of the spatial Hill's problem. Dyn. Syst., 20(1):115-147, 2005.

[13] G. Gómez and J. M. Mondelo. The dynamics around the collinear equilibrium points of the RTBP. Phys. D, 157(4):283-321, 2001.

[14] K. C. Howell, B. T. Barden, R. S. Wilson, and M. W. Lo. Trajectory design using a dynamical systems approach with application to GENESIS. Advances in the Astronautical Sciences, 97:1665-1684, 1998.

[15] A. Jorba and J. J. Masdemont. Dynamics in the center manifold of the Restricted Three-Body Problem. Physica D, 132:189-213, 1999.

[16] A. Jorba and M. Zou. A software package for the numerical integration of odes by means of high-order Taylor methods. Experimental Mathematics, 14(1):99-117, 2005.

[17] O. Yu Koltsova, L. H. Lerman, Periodic and homoclinic orbits in a two parameter unfolding of a Hamiltonian system with a homoclinic orbit to a saddle-center, Intern. J. of Bif. and Chaos, 5(2): 397-408, 1995.

[18] W. S. Koon, M. W. Lo, J. E. Marsden, and S. D. Ross. Heteroclinic connections between periodic orbits and resonance transitions in celestial mechanics. Chaos, 10(2):427-469, 2000.

[19] J. Llibre, R. Martínez, and C. Simó. Tranversality of the invariant manifolds associated to the Lyapunov family of periodic orbits near $L_{2}$ in the restricted three-body problem. J. Differential Equations, 58(1):104-156, 1985.

[20] R. P. McGehee. Some homoclinic orbits for the restricted three-body problem. PhD thesis, University of Wisconsin, 1969. 
[21] K. R. Meyer and G. R. Hall. Introduction to Hamiltonian dynamical systems and the N-body problem, volume 90 of Applied Mathematical Sciences. Springer-Verlag, New York, 1992.

[22] T. Sweetser, S. Broschart, V. Angelopoulos, G. Whiffen, D. Folta, M.-K. Chung, S. Hatch, and M. Woodard. ARTEMIS mission design. Space Sci Rev, 165:27-57, 2011.

[23] V. Szebehely. Theory of Orbits. The Restricted Problem of Three Bodies. Academic Press, Inc., 1967.

[24] D. Wilczak and P. Zgliczyński. Heteroclinic connections between periodic orbits in planar restricted circular three-body problem-a computer assisted proof. Comm. Math. Phys., 234(1):37-75, 2003.

[25] D. Wilczak and P. Zgliczyński. Heteroclinic connections between periodic orbits in planar restricted circular three body problem. II. Comm. Math. Phys., 259(3):561-576, 2005. 